\title{
Expression of cdc25A and cdc25B proteins in thyroid neoplasms
}

\author{
Y Ito*,', H Yoshida', K Nakano', K Kobayashi', T Yokozawa', K Hirai', F Matsuzuka', N Matsuura², K Kakudo³, \\ K Kuma' and A Miyauchi'
}

'Department of Surgery, Kuma Hospital, 8-2-35, Shimoyamate-dori, Chuo-ku, Kobe City 650-00 II, Japan; ²Department of Pathology, School of Allied Health Science, Osaka University Faculty of Medicine, I-7, Yamadaoka, Suita, Osaka 565-087I, Japan; ${ }^{3}$ Department of Pathology, Wakayama Medical College,

Kimiidera 8I I-I, Wakayama City, 64 I-0012, Japan

Cdc25B and cdc25A phosphates are prominent stimulators of cell cycle progression and recent studies have also suggested their oncogenic roles. To elucidate the role of these proteins in thyroid neoplasms, we immunohistochemically investigated their expression, and neither protein was expressed in normal follicular cells. Cdc25B was frequently overexpressed in follicular adenoma and minimally invasive follicular carcinoma, but the incidence was significantly lower in widely invasive follicular carcinoma. Furthermore, the $c d c 25 B$ expression level significantly decreased with the dedifferentiation of thyroid carcinoma. Cdc25A overexpression was observed in high incidences in all types of thyroid neoplasms. These results suggest that cdc $25 \mathrm{~B}$ and cdc25A play oncogenic roles in thyroid follicules and that cdc25B works predominantly in the early phase of the progression of thyroid carcinoma, whereas cdc25A plays a fundamental role in the development of thyroid neoplasms. British Journal of Cancer (2002) 86, 1909 - 1913. doi: I0.1038/sj.bjc.6600364 www.bjcancer.com

(c) 2002 Cancer Research UK

Keywords: cdc25A; cdc25B; thyroid tumour; immunohistochemistry

Thyroid carcinoma is one of the most common malignancies originating from the endocrine organs. Two types of carcinoma are known to occur from normal follicular cells, which are papillary and follicular carcinomas. Follicular carcinoma is said to arise from preexisting follicular adenoma, although this has not yet been confirmed. Precursor lesions of papillary carcinoma, on the other hand, have not yet been identified (Faggin, 2000). It is well known that the biological characteristics of these carcinomas are generally mild and the prognosis is excellent if operated on competently. However, anaplastic carcinoma, also known as undifferentiated carcinoma, arises from papillary or follicular carcinoma and is one of the most aggressive human carcinomas with a dire prognosis. Although many kinds of therapeutic strategy have been performed, most patients die within 6 months after diagnosis (Aldinger et al, 1978). Furthermore, Sakamoto et al (1983) demonstrated that papillary or follicular carcinoma with a solid, trabecular or scirrhous growth pattern showed a worse clinical outcome than pure papillary or follicular carcinoma. They proposed a clinicopathological entity of poorly differentiated carcinoma, and hypothesised that this type of carcinoma falls between well differentiated carcinoma and undifferentiated carcinoma, although it is still an open question whether showing such growth patterns is truly due to the dedifferentiation of carcinoma.

To evaluate the biological aggressiveness, one prominent factor is the cell proliferating activity, which reflects the cell cycle progression. In the cell cycle, two checkpoints have been identified, which are located in the G1-S phase and the G2-M phase. The complexes of various cyclins and cyclin dependent kinases (CDKs) play a crucial role in exceeding these checkpoints and CDKs should be activated by phosphorylation in order to work as positive regula-

*Correspondence: Y Ito; E-mail: ito0 I@kuma-h.or.jp

Received I8 December 200 I; revised 9 April 2002; accepted II April 2002 tors of the cell cycle (Sherr, 1994). Two kinds of phosphatase, cdc25A and cdc25B are known to active CDKs, resulting in positive regulation of the cell cycle progression (Galactinov and Beach, 1991). As cdc25A mRNA expression is elevated in the late G1 phase and the microinjection of a specific antibody against cdc25A blocks G1-S transition, it is suggested that cdc25A activates the CDKs, making a complex with the G1 cyclins (Jinno et al, 1994). On the other hand, cdc25B plays a crucial role in G2-M transition, of which the target should be cyclin B1/cdc2 complex (Lammer et $a l, 1998)$. Furthermore, cdc25A and cdc25B can induce a malignant transformation in rodent cells in cooperation with either $\mathrm{Ha}$ RASG12V or loss of RB1, indicating that they are potential oncogenes (Galactinov et al, 1995).

The expression of $\mathrm{cdc} 25 \mathrm{~A}$ and $\mathrm{cdc} 25 \mathrm{~B}$ has been studied in some human neoplasms and various results have been obtained (Kudo et al, 1997; Wu et al, 1998; Broggini et al, 2000; Takemasa et al, 2000; Nishioka et al, 2001; Sasaki et al, 2001). In thyroid carcinomas, studying the modulation of cell proliferation is also important, because the activity of cell proliferation strongly reflects the biological aggressiveness of this carcinoma (Erickson et al, 1998). In this study, therefore, we investigated the expression of cdc25A and cdc25B in order to elucidate the role of these proteins as modulators of the cell cycle progression of this carcinoma.

\section{MATERIALS AND METHODS}

\section{Tissue specimens}

Ten per cent formalin-fixed, paraffin-embedded blocks were prepared from the surgical specimens of 172 cases of thyroid tumours. They consisted of 19 follicular adenomas, 26 minimally invasive follicular carcinomas, 23 widely invasive follicular carcinomas, 72 cases of papillary carcinomas, and 32 cases of anaplastic (undifferentiated) carcinomas. This project was approved by the Ethics Committees of Kuma Hospital. 


\section{Antibodies}

Polyclonal antibodies against cdc25B (sc-326) and cdc25A (sc-97) were purchased from Santa Cruz Biotechnology (Santa Cruz, CA, USA). They were applied as primary antibodies at a concentration of $1: 200$ and $1: 400$, respectively.

\section{Immunohistochemistry}

Tissue sections $4 \mu \mathrm{m}$ thick were dewaxed and endogenous peroxidase activity was blocked with $0.3 \%$ hydrogen peroxide in methanol for $15 \mathrm{~min}$. After rinsing in distilled water, the sections were then immersed in $0.03 \mathrm{~mol} \mathrm{~L}^{-1}$ citrate buffer ( $\mathrm{pH} \mathrm{6.0)}$ ) and incubated at $95^{\circ} \mathrm{C}$ for $40 \mathrm{~min}$ in a water bath for antigen retrieval. After rinsing in phosphate-buffered saline pH 7.2 (PBS), $10 \%$ bovine serum (Wako, Osaka, Japan) was applied for 20 min to block nonspecific reactions. Sections were then incubated with the primary antibody overnight at $4^{\circ} \mathrm{C}$. After rinsing in PBS, they were treated with peroxidase-labelled anti-rabbit immunoglobulins (Nichirei, Tokyo, Japan) for $30 \mathrm{~min}$. The peroxidase reaction was visualised by incubating the sections with $0.02 \% 3,3^{\prime}$-diaminobenzidine tetrahydrochloride in $0.05 \mathrm{M}$ Tris buffer with $0.01 \%$ hydrogen peroxide (Nichirei, Tokyo, Japan). The sections were counterstained with haematoxylin. Sections for the negative control were prepared using rabbit immunoglobulins instead of the primary antibody.

\section{Immunohistochemical evaluation}

We regarded the cells as positive for these proteins when their immunoreactivity was clearly observed in their nuclei or cytoplasms, or in both. We classified the cases into four categories according to their positive cell rate as follows: $(-),<10 \%,(+)$, from 10 to $25 \%$, (++), from 25 to $75 \%,(+++),>75 \%$. We regarded the cases classified as $(++)$ or $(+++)$ as overexpressing these proteins.

\section{Statistical analyses}

We employed Fischer's exact test for analyses of the relationship between the expression of these proteins and their histological types. $P$ values less than 0.05 were regarded as statistically significant.

\section{RESULTS}

\section{Expression of cdc25B}

Cdc25B was not expressed in normal follicular cells or stromal cells, including lymphocytes and epithelial cells of the blood vessels (not shown), whereas it was predominantly expressed in the cytoplasms of tumour cells. In follicular tumours, cdc25B overexpression was observed in 41 of the 68 cases $(60.3 \%)$ (Figure 1A). In particular, follicular adenoma and minimally invasive follicular carcinoma very frequently overexpressed cdc25B, 63.2\% (12 of the 19 cases) and $73.1 \%$ (19 of the 26 cases), respectively. In widely invasive follicular carcinoma, this phenomenon was seen in only $43.5 \%$ ( 10 of the 23 cases) of the cases (Figure 1B), which was significantly lower $(P=0.0456)$ than that in minimally invasive carcinoma (Table 1).

In papillary carcinoma, 44 of the 72 cases $(61.1 \%)$ overexpressed cdc25B (Figure 1C), indicating that this phenomenon was observed in 74 of the 121 cases $(61.2 \%)$ of papillary or follicular carcinoma. Among these cases, 43 had lesions showing a solid, trabecular or scirrhous growth pattern, which was consistent with the criteria of poorly differentiated carcinoma proposed by Sakamoto et al (1983) (Figure 1C). Table 2 shows the relationship between cdc25B overexpression and carcinoma differentiation. The incidence of
cdc25B overexpression in well differentiated carcinoma was $74.4 \%$ (58 of the 78 cases). It was significantly higher than that in poorly differentiated carcinoma $(P<0.0001)$, which was $37.2 \%$ (16 of the 43 cases). In undifferentiated carcinoma, the incidence was only $9.3 \%$ (three of the 32 cases) (Figure 1D), which was lower than in poorly differentiated carcinoma $(P=0.0072)$. Thus, the reduced expression of $\mathrm{cdc} 25 \mathrm{~B}$ was significantly linked to dedifferentiation of thyroid carcinoma $(P<0.0001)$.

\section{Expression of cdc25A}

Cdc25A expression was not observed in normal follicular cells or stromal cells (not shown). In thyroid neoplasms, its immunoreactivity was localised in the nuclei or cytoplasms or in both. One hundred and five cases of 153 thyroid carcinomas (68.7\%) (Figure $2 \mathrm{~A}, \mathrm{~B})$ and six cases of 19 adenomas (31.6\%) overexpressed cdc25A (Figure 2C). Among them, cdc25A was localised predominantly in the cell nuclei in 29 carcinomas $(27.6 \%)$ (Figure 2B) and three adenomas $(50 \%)$, in the cytoplasms in 66 carcinomas $(62.9 \%)$ (Figure 2A) and two adenomas (33.3\%), and in the remaining 10 carcinomas $(9.5 \%)$ and one adenoma (16.7\%) (Figure $2 \mathrm{C}$ ), it was expressed both in the nuclei and cytoplasms in similar incidences. We investigated the relationship between the cell localisation of cdc25A and histological types, but no statistically significant relationships were established (not shown).

We investigated the relationship between cdc25A expression and clinicopathological features, as shown in Tables 3 and 4, although we could not establish any significance. Furthermore, no relationships were determined between the expression of cdc25A and cdc25B in any histological types (data not shown).

\section{DISCUSSION}

In this study, we demonstrated that (1) cdc25B and cdc25A were not overexpressed in normal thyroid tissue, (2) cdc25B was frequently overexpressed in follicular adenoma and minimally invasive follicular carcinoma, but the incidence significantly decreased in widely invasive follicular carcinoma, (3) the incidence of cdc25B overexpression significantly decreased with the dedifferentiation of carcinoma, and (4) cdc25A was frequently overexpressed in thyroid neoplasms regardless of their histological types.

A difference in cell localisation between these two phosphatases has been reported. Previous in vitro and clinical studies indicated the cytoplasmic accumulation of cdc25B, which is in agreement with our findings (Gabrielli et al, 1996; Gasparotte et al, 1997; Kudo et al, 1997; Broggini et al, 2000; Takemasa et al, 2000; Nishioka et al, 2001). On the other hand, Dixon et al (1998) showed an elevated expression of cdc25A in nuclear fractions of colon carcinoma, which was confirmed by immunohistochemical study (Takemasa et al, 2000). However, in oesophageal carcinoma, similar to our results, cdc25A was accumulated both in cytoplasms and nuclei (Nishioka et al, 2001), and in ovarian carcinoma, its immunoreactivity was seen mainly in cytoplasms (Broggini et al, 2000), indicating that the localisation of this phosphatase depends on the origin of the carcinoma.

What is most interesting in this study is the inverse relationship between cdc25B overexpression and the biological aggressiveness of the thyroid tumour, because such an expression status has not been observed in other positive regulators of cell cycle. Wang et al (2000) demonstrated that cyclin D1 was more frequently overexpressed in more aggressive thyroid carcinomas such as anaplastic carcinoma, tall cell variant, and insular carcinoma. On the other hand, Goto et al (2001) showed that cyclin D1 was frequently overexpressed in thyroid carcinoma but rarely in benign adenoma. Furthermore, our recent study showed that cyclin A expression level increased with dedifferentiation of thyroid carcinoma and cyclin B1 overexpression was found exclusively in undifferentiated carcinoma (manuscript submitted). It is thus suggested that the 
A

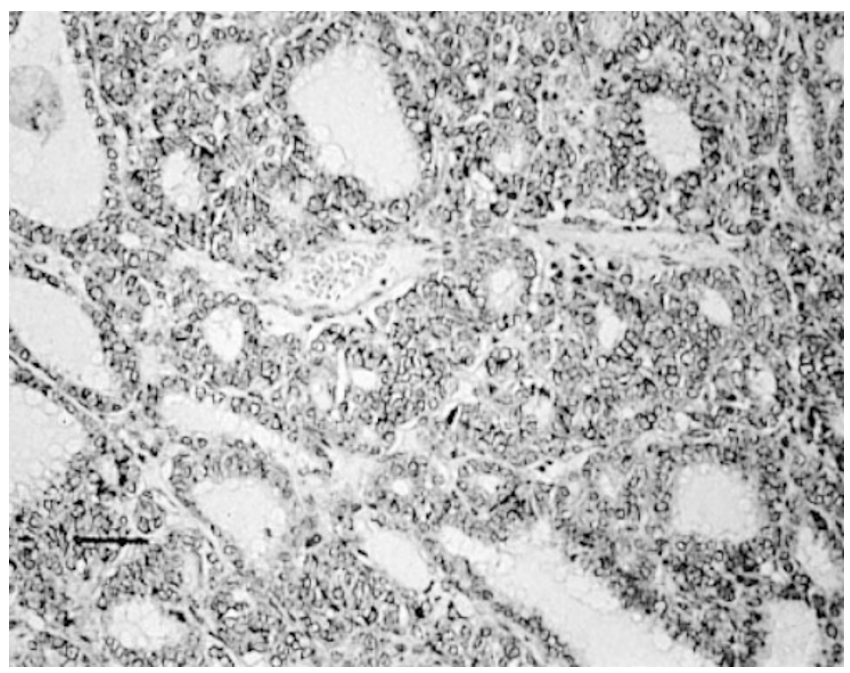

C

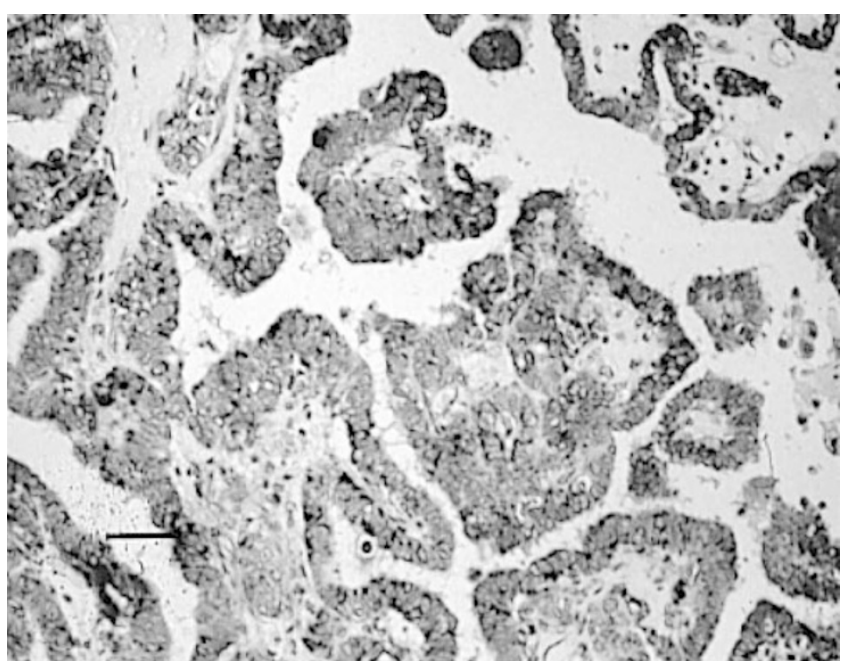

B

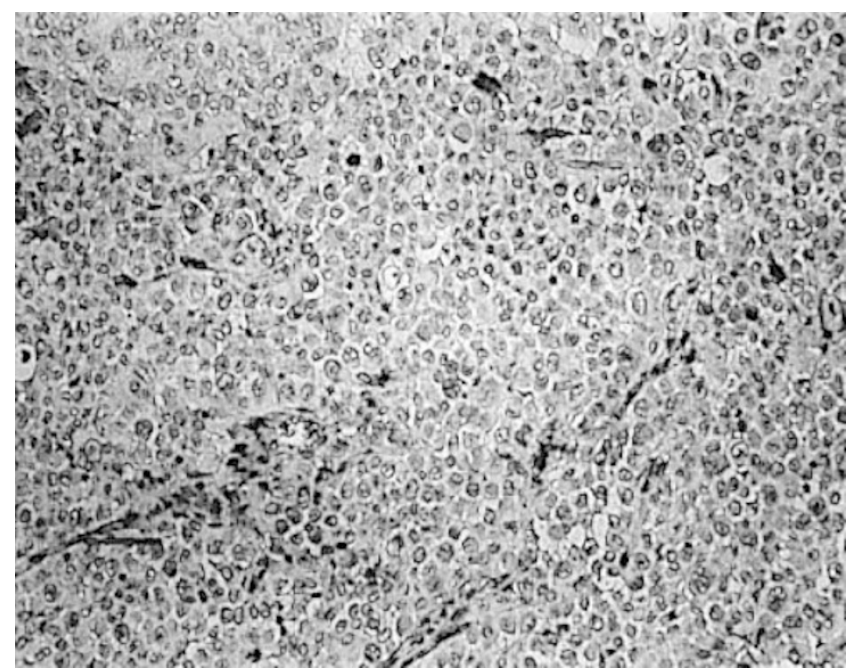

\section{D}

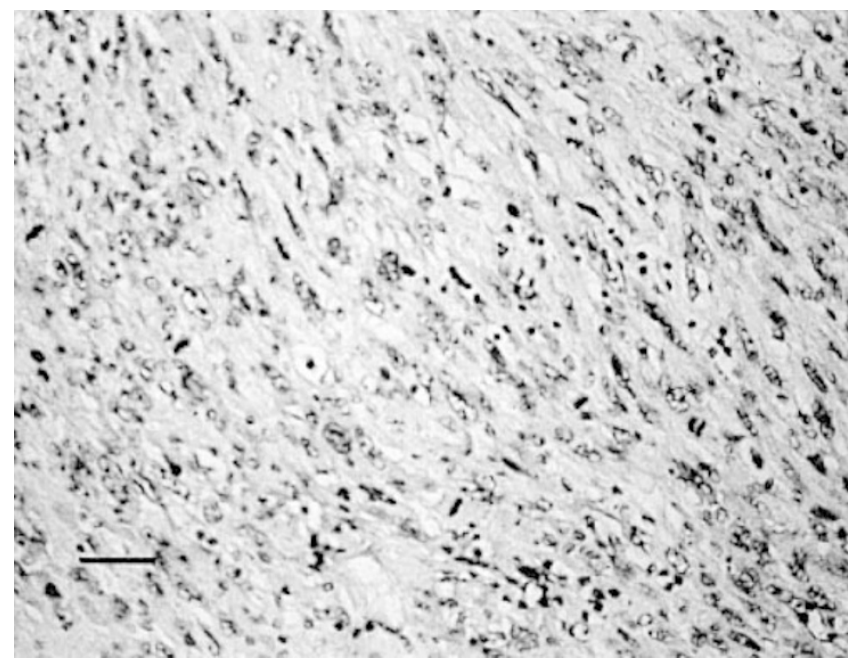

Figure I Immunostaining of cdc25B. (A) cdc25B overexpression in minimally invasive follicular carcinoma (+++). (B) cdc25B overexpression in widely invasive follicular carcinoma with solid growth pattern $(++) .(\mathbf{C})$ cdc25 overexpression in papillary carcinoma $(+++)$. (D) cdc25 was negative in undifferentiated carcinoma $(-)$.

Table I Relationship between cdc25B overexpression and histological type of follicular tumour

\begin{tabular}{llrl}
\hline Cdc25B overexpression & + & - & Total \\
\hline Follicular carcinoma & & & \\
*Widely invasive & 10 & 13 & 23 \\
*Minimally invasive & 19 & 7 & 26 \\
Follicular adenoma & 12 & 7 & 19 \\
Total & 41 & 27 & 68 \\
\hline
\end{tabular}

* $P=0.0456$ (Fisher's exact test).

reduced expression of cdc25B in dedifferentiated carcinoma is unique compared to other cell cycle regulatory proteins.

The expression status of cdc25B in thyroid tumours differs from that in other carcinomas. Previous studies have demonstrated that cdc25B overexpression reflects a worse clinical outcome in patients with colorectal (Takemasa et al, 2000), ovarian (Broggini et al, 2000), and non-small-cell lung carcinomas (Sasaki et al, 2001). In gastric carcinoma, cdc25B expression was associated with advanced stage and deep invasion (Galactinov et al, 1995).
Table 2 Relationship between cdc25B overexpression and the differentiation of thyroid carcinoma

\begin{tabular}{lrrr}
\hline Cdc25B overexpression & $\boldsymbol{+}$ & - & Total \\
\hline Undifferentiated carcinoma & 3 & 29 & 32 \\
Papillary or follicular carcinoma & 74 & 47 & 121 \\
$\quad$ With solid, trabecular or scirrhous growth pattern & 16 & 27 & 43 \\
$\quad \begin{array}{l}\quad \text { (poorly differentiated) } \\
\quad \text { (well differentiated) }\end{array}$ & 58 & 20 & 78 \\
Total & 77 & 76 & 153 \\
\hline
\end{tabular}

$P<0.0001$ (undifferentiated carcinoma vs poorly differentiated carcinoma vs well differentiated carcinoma). $P<0.0001$ (well differentiated carcinoma vs poorly differentiated carcinoma). $P=0.0072$ (undifferentiated carcinoma vs poorly differentiated carcinoma)

However, the physiological roles of cdc25B in carcinomas do not appear to be simple. Takemasa et al (2000) demonstrated that, although cdc25B overexpression can be regarded as an independent prognostic factor in colorectal carcinoma, it is not related to the cell proliferating activity evaluated by the Ki-67 labelling index. 
Table 3 Relationship between cdc25A overexpression and histological type of follicular tumour

A

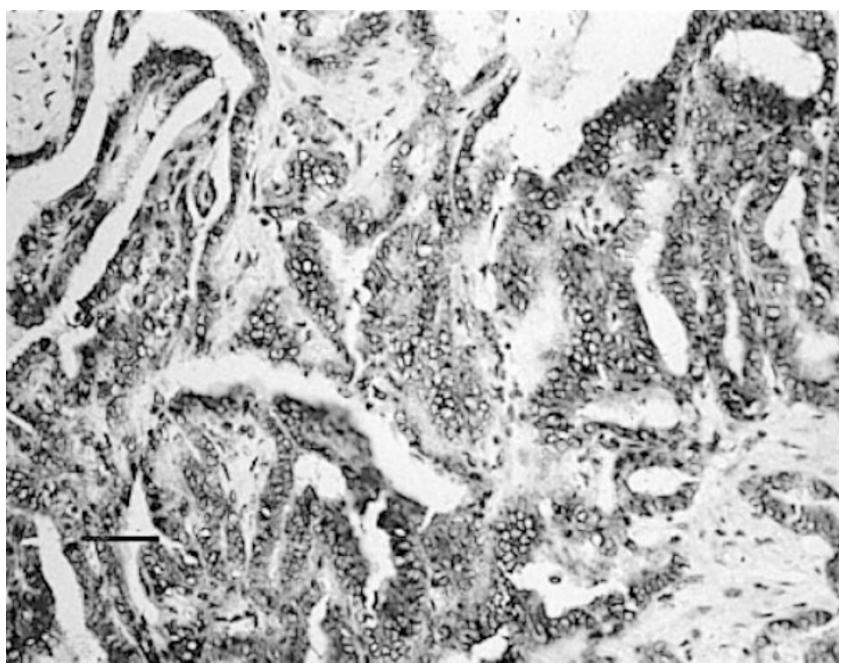

B
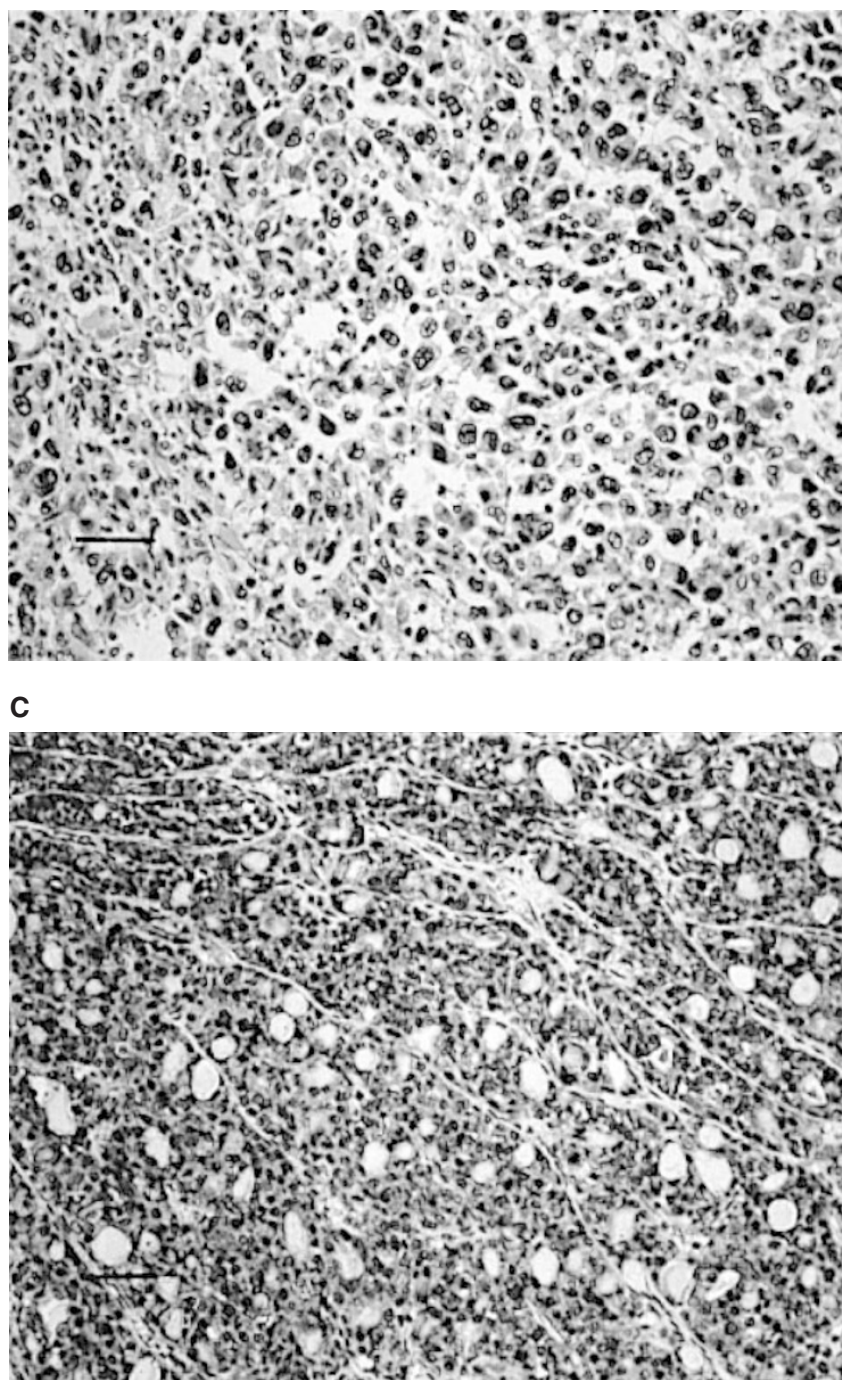

Figure 2 Immunostaining of cdc25A. (A) cdc25A overexpression in cy toplasms of papillary carcinoma $(+++)$. (B) cdc25A overexpression in cel nuclei of undifferentiated carcinoma $(+++)$. (C) cdc25A overexpression in cytoplasms and cell nuclei of follicular adenoma (+++). Scale bars: I50 $\mu \mathrm{m}$.

\begin{tabular}{lrrr}
\hline Cdc25A overexpression & + & - & Total \\
\hline Follicular carcinoma & & & \\
Widely invasive & 13 & 10 & 23 \\
Minimally invasive & 13 & 13 & 26 \\
Follicular adenoma & 6 & 13 & 19 \\
Total & 42 & 26 & 68 \\
\hline
\end{tabular}

Not significant.

Table 4 Relationship between cdc25A overexpression and the differentiation of thyroid carcinoma

\begin{tabular}{lccc}
\hline Cdc25A overexpression & $\mathbf{+}$ & - & Total \\
\hline Undifferentiated carcinoma & 17 & 15 & 32 \\
Papillary or follicular carcinoma & 88 & 33 & 121 \\
$\quad$ With solid, trabecular or scirrhous growth pattern & 34 & 9 & 43 \\
$\quad$ (poorly differentiated) & 54 & 24 & 78 \\
$\begin{array}{l}\text { Pure papillary or follicular carcinoma } \\
\quad \text { well differentiated) }\end{array}$ & 105 & 48 & 153 \\
$\begin{array}{l}\text { Total } \\
\text { P }\end{array}$ & & &
\end{tabular}

Not significant.

These findings are strange, because cdc25B fundamentally acts as a positive regulator of cell cycle progression. They thus hypothesised that cdc25B itself displays oncogenic properties by enhancing the malignant nature of this carcinoma. Also, in thyroid carcinoma, the clinical significance of $\operatorname{cdc} 25 \mathrm{~B}$ overexpression is very complicated. Previous studies have demonstrated that, in thyroid neoplasms, cell proliferating activity is usually low, except for its drastic elevation in undifferentiated carcinoma (Erickson et al, 1998). This study showed the frequent overexpression of cdc25B in benign adenoma and carcinomas with low aggressive phenotypes and its decreased expression in those of very aggressive types, such as undifferentiated carcinoma and widely invasive follicular carcinoma, indicating that cdc25B expression is even inversely linked to the cell proliferating activity of thyroid neoplasms. It is thus suggested that $\operatorname{cdc} 25 \mathrm{~B}$ plays a crucial role in the progression of thyroid carcinoma in the early stage, as well as in the tumorigenesis of follicular cells of the thyroid, rather than merely in tumour cell proliferation. This protein does not seem to be necessary for the development of thyroid carcinoma after it achieves high proliferating activity, including dedifferentiation.

The clinical significance of cdc25A in carcinoma also seems to vary, because different results have been reported for carcinomas of different origin (Broggini et al, 2000; Takemasa et al, 2000; Nishioka et al, 2001). In oesophageal and ovarian carcinomas, its overexpression significantly predicts a poor prognosis whereas no such relation could be established in colorectal carcinoma. According to our findings, cdc25A was frequently overexpressed in all types of thyroid neoplasms including benign adenoma arising from follicular cells. Unlike cdc25B, the incidence of cdc25A overexpression did not decrease in carcinomas with aggressive phenotypes. It is therefore suggested that this phosphatase plays a fundamental role in the oncogenesis of thyroid follicles and also in the development of thyroid neoplasms, regardless of histological type. Previous studies have shown that the overexpression of G1 cyclins could be observed in thyroid neoplasms of various types in similar incidences (Wang et al, 2000; Goto et al, 2001). Thus, we can hypothesise that $\operatorname{cdc} 25 \mathrm{~A}$ may be linked to the activation of cdks, making complexes with cyclins, although it is unlikely that both cdc25A and cdk-G1 cyclin complex are directly related to the cell proliferating activity of thyroid neoplasms. 
The significant reduction of cdc25B expression in dedifferentiated thyroid carcinoma has prompted researchers to investigate its prognostic value. Of the 32 undifferentiated carcinomas in our series, only 17 cases could undergo curative surgery. Among them, one case which overexpressed cdc25B has survived for 6 months after surgery, whereas the three long term survival cases, 82,56 , and 44 months, did not overexpress cdc25B. We therefore failed to establish the prognostic value of cdc25B expression, but

\section{REFERENCES}

Aldinger KA, Samaan NA, Ibanez M, Hill Jr CS (1978) Anaplastic carcinoma of the thyroid: a review of 84 cases of spindle and giant-cell carcinoma of the thyroid. Cancer 41: 2267-2275

Broggini M, Buraggi G, Brenna A, Riva L, Codegoni AM, Torri V, Lissoni AA, Mangioni C, D’Incalci M (2000) Cell cycle-related phosphatases CDC25A and $\mathrm{B}$ expression correlates with survival in ovarian cancer patients. Anticancer Res 20: $4835-4840$

Dixon D, Moyana T, King MJ (1998) Elevated expression of the cdc25A protein phosphatase in colon cancer. Exp Cell Res 240: 236-243

Erickson LA, Jin L, Wollan PC, Thompson GB, van Heerden J, Lloyd RB (1998) Expression of p27kip1 and Ki-67 in benign and malignant thyroid tumors. Mod Pathol 11: 169-174

Faggin JA (2000) Molecular genetics of tumors of thyroid follicular cells. In. The Thyroid, 8th ed, Braverman LE, Utiger RD (eds) pp 886-898 Lippincott Williams \& Wilkins

Gabrielli BG, De Souza CP, Toonks ID, Clark JM, Hayward NK, Ellem KA (1996) Cytoplasmic accumulation of cdc25B phosphatase in mitosis triggers centrosomal mucrotubule nucleation in HeLa cells. J Cell Sci 109: $1081-1093$

Galactinov K, Beach D (1991) Specific activation of cdc25 tyrosine phosphatases by B-type cyclins: evidence for multiple roles of mitotic cyclins. Cell 67: $1181-1194$

Galactinov K, Lee AK, Eckstein J, Draetta G, Meckler J, Loda M, Beach D (1995) CDC25 phosphatases as potential human oncogenes. Science (Washington DC) 269: 1575-1577

Gasparotto D, Maestro R, Piccinin S, Vukosavljevic T, Barzan L, Sulfaro S, Boiocchi M (1997) Overexpression of CDC25A and CDC25B in head and neck cancers. Cancer Res 57: 2366-2368

Goto A, Sakamoto A, Machinami R (2001) An immunohistochemical analysis of cyclin D1, p53, and p21 WAF1/CIP1 proteins in tumors originating from the follicular epithelium of the thyroid gland. Pathol Res Pract 197: $217-222$ further studies with a larger number of cases undergoing curative surgery are necessary to draw a definitive conclusion about this point.

In summary, this study demonstrated that $\operatorname{cdc} 25 \mathrm{~B}$ and $\operatorname{cdc} 25 \mathrm{~A}$ may play an oncogenic role in thyroid neoplasms but may not be directly linked to the cell proliferation of thyroid tumours. Further studies are necessary to more clearly elucidate the significance of these proteins in thyroid neoplasms.

Jinno S, Suto K, Nagata A, Igarashi M, Kanaoka Y, Nojima H, Okayama H (1994) Cdc25A is a novel phosphatase functioning early in the cell cycle. EMBO J 13: $1549-1556$

Kudo Y, Yasui W, Ue T, Yamamoto S, Yokozaki H, Nikai H, Tahara E (1997) Overexpression of cyclin-dependent kinase-activating CDC25B phosphatase in human gastric carcinomas. Jpn J Cancer Res 88: $947-952$

Lammer C, Wagerer S, Saffrich R, Mertens D, Ansorge W, Hoffmann I (1998) The cdc25B phosphatase is essential for the G2/M phase transition in human cells. J Cell Sci 111: 2445-2553

Nishioka K, Doki Y, Shiozaki H, Yamamoto H, Tamura S, Yasuda T, Fujiwara Y, Yano M, Miyata H, Kishi K, Nakagawa H, Shamma A, Monden M (2001) Clinical significance of CDC25A and CDC25B expression in squamous cell carcinomas of the oesophagus. Br J Cancer 85: $412-421$

Sakamoto A, Kasai N, Sugano H (1983) Poorly differentiated carcinoma of the thyroid. A clinicopathological entity for a high-risk group of papillary and follicular carcinomas. Cancer 52: 1849-1855

Sasaki H, Yukiue H, Kobayashi Y, Tanahashi M, Moriyama S, Nakashima Y, Fukai I, Kiriyama M, Yamakawa Y, Fujii Y (2001) Expression of the cdc25B gene as a prognosis marker in non-small cell lung cancer. Cancer Lett 173: $187-192$

Sherr CJ (1994) Mammalian G1 cyclins. Cell 79: $551-555$

Takemasa I, Yamamoto H, Mitsugu S, Ohur M, Noura S, Miyake Y, Matsumoto T, Aihara T, Tomita N, Tamaki Y, Sakita I, Kikkawa N, Matsuura N, Shiozaki H, Monden M (2000) Overexpression of CDC25B phosphatase as a novel marker of poor prognosis of human colorectal carcinoma. Cancer Res 60: $3043-3050$

Wang S, Lloyd RV, Hutzler MJ, Safran MS, Patwardhan NA, Khan A (2000) The role of cell cycle regulatory protein, cyclin D1, in the progression of thyroid cancer. Mod Pathol 13: $882-887$

Wu W, Fan YH, Kemp BL, Walsh G, Mao L (1998) Overexpression of cdc25A and cdc $25 \mathrm{~B}$ is frequent in primary non-small cell lung cancer but is not associated with overexpression of c-myc. Cancer Res 58: $4082-4085$ 